Интернет-журнал «Транспортные сооружения» / Russian journal of transport engineering http://t-s.today/

2016, Том 3, №4 / 2016, Vol 3, No 4 http://t-s.today/issues/vol3-no4.html

URL статьи: http://t-s.today/PDF/04TS416.pdf

DOI: 10.15862/04TS416 (http://dx.doi.org/10.15862/04TS416)

Ссылка для цитирования этой статьи:

Муравьева Л.В. Влияние изменчивости касательных напряжений в теле трубы морского подводного трубопровода с бетонным покрытием при сейсмическом воздействии // Интернет-журнал «Транспортные сооружения», Том 3, №4 (2016) http://t-s.today/PDF/04TS416.pdf (доступ свободный). Загл. с экрана. Яз. рус., англ. DOI: 10.15862/04TS416

For citation:

Muravyeva L.W. [The impact of the shear stress change in the pipe body of sea-line with a concrete coating under a seismic load] Russian journal of transport engineering, 2016, Vol. 3, no. 4. Available at: http://t-s.today/PDF/04TS416.pdf (In Russ.) DOI: 10.15862/04TS416

\title{
УДК 629.039.58
}

Муравьева Людмила Викторовна ФГАОУ ВО «Санкт-Петербургский государственный политехнический университет», Россия, Санкт-Петербург Профессор кафедры «Водохозяйственное и гидротехническое строительство» Доктор технических наук

E-mail: rfludmia@yandex.ru

\section{Влияние изменчивости касательных напряжений в теле трубы морского подводного трубопровода с бетонным покрытием при сейсмическом воздействии}

Аннотация. Толстостенная оболочка морского трубопровода, испытывает действие сочетание воздействий от эксплуатационных нагрузок (давления, температуры, вес бетонного покрытия), при учете вероятности возникновения вибрационных, сейсмических нагрузок. Оценку прочности толстостенной оболочки газопровода необходимо проводить на основании проверки на устойчивость при комбинированном воздействии изгиба и наружного давления. Целью работы является исследование особенностей напряженно-деформируемого состояния участков морского трубопровода с бетонным покрытием при сейсмических воздействиях.

Ключевые слова: толстостенная оболочка; бетонное покрытие; касательная сила; сейсмостойкость.

В процессе эксплуатации толстостенная оболочка морского трубопровода, расположенного на дне моря, испытывает равномерно распределенные вокруг ее оси внутреннее давление транспортируемого продукта и наружное гидростатическое давление окружающей жидкости, определяемое глубиной моря.

Задача определения напряженно-деформированного состояния оболочки трубопровода при действии эти нагрузок решена в работах [1,7], исходя из симметрии оболочки и действующих нагрузок.

В результате решения имеем: 


$$
\begin{aligned}
\sigma_{r} & =\frac{r_{1}^{2} p-r_{2}^{2} q}{r_{2}^{2}-r_{1}^{2}}+\frac{r_{1}^{2}(q-p)}{r_{2}^{2}-r_{1}{ }^{2}} \frac{r_{2}^{2}}{r^{2}} \\
\sigma_{\theta} & =\frac{r_{1}^{2} p-r_{2}^{2} q}{r_{2}^{2}-r_{1}{ }^{2}}-\frac{r_{1}^{2}(q-p)}{r_{2}^{2}-r_{1}^{2}} \frac{r_{2}^{2}}{r^{2}} \\
\sigma_{x} & =2 v \frac{r_{1}^{2} p-r_{2}^{2} q}{r_{2}^{2}-r_{1}{ }^{2}}
\end{aligned}
$$

Радиальное напряжение равно окружному (рис. 1) в любой точке цилинра с большой толщиной стенки. При отсутствии осевых напряжений весь материал цилиндра находится в состоянии чистого сдвига [1].

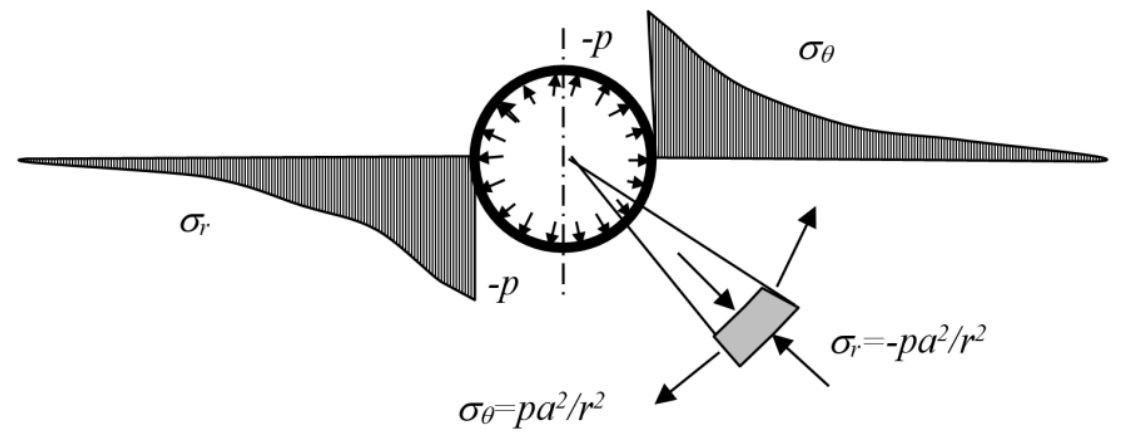

Рисунок 1. Цилиндр с бесконечно большой толщиной стенки (рисунок автора)

Figure 1. The cylinder with an infinitely large thickness of wall (the author's drawing)

Напряжения находятся в обратно пропорциональной зависимости от квадрата радиуса $r$, в точках, на расстоянии $r=4 a$ от оси, напряжения составляют 1/16 от максимальных.

При изменении Значение внутреннего давления, то распределения напряжений от радиального $\sigma_{r}$ и кольцевого $\sigma_{\theta}$ по толщине $\mathrm{r}$ стенки толстостенной оболочки будут продвигаться к внешней поверхности трубы (2).

Эквивалентное напряжение в произвольной точке трубопровода равно

$$
\sigma_{i}=\frac{1}{\sqrt{2}} \sqrt{\left(\sigma_{r}-\sigma_{\theta}\right)^{2}+\left(\sigma_{\theta}-\sigma_{x}\right)^{2}+\left(\sigma_{x}-\sigma_{r}\right)^{2}}=\frac{q r_{2}^{2}}{r_{2}^{2}-r_{1}^{2}} \sqrt{3 \frac{r_{1}^{4}}{r^{4}}+(1-2 v)^{2}}
$$

здесь $x, r, \theta$, главные оси напряженного состояния. [1]

Ограниченность статистических данных по механическим свойствам, которые используются при строительстве морских подводных трубопроводов, требует выполнения математического моделирования, экспериментальных исследований натурных образцов труб. Такие работы проводились при строительстве глубоководных трубопроводов (Штокмановского месторождения, Голубой поток).

Исследования $[1 ; 3 ; 4]$ показали, что при увеличении соотношения D/ $\delta$ удельное влияние изгибающего момента все более возрастает, и вместо составления диаграммы давление изгибающий момент необходимо использовать систему двух независимых условий

$$
\left\{\begin{array}{l}
p \leq p_{c} \\
M \leq M_{c}
\end{array}\right.
$$

В сечении, где изгибающий момент достигает максимального значения, возможно выпучиване оболочки. 
Потере устойчивости цилиндрической формы равновесия оболочки трубопровода способствует и гидростатическое давление морской воды q над газопроводом и внешние сейсмическое воздействие. Продольные сжимающие напряжения в стенке оболочки $\sigma_{x}=2 v \frac{r_{2}^{2}}{r_{2}^{2}-r_{1}^{2}} q$ суммируются с напряжениями от изгиба $\sigma_{\max }= \pm \frac{q l^{2}}{8 W}$, в сжатой области оболочки (рис. 2).

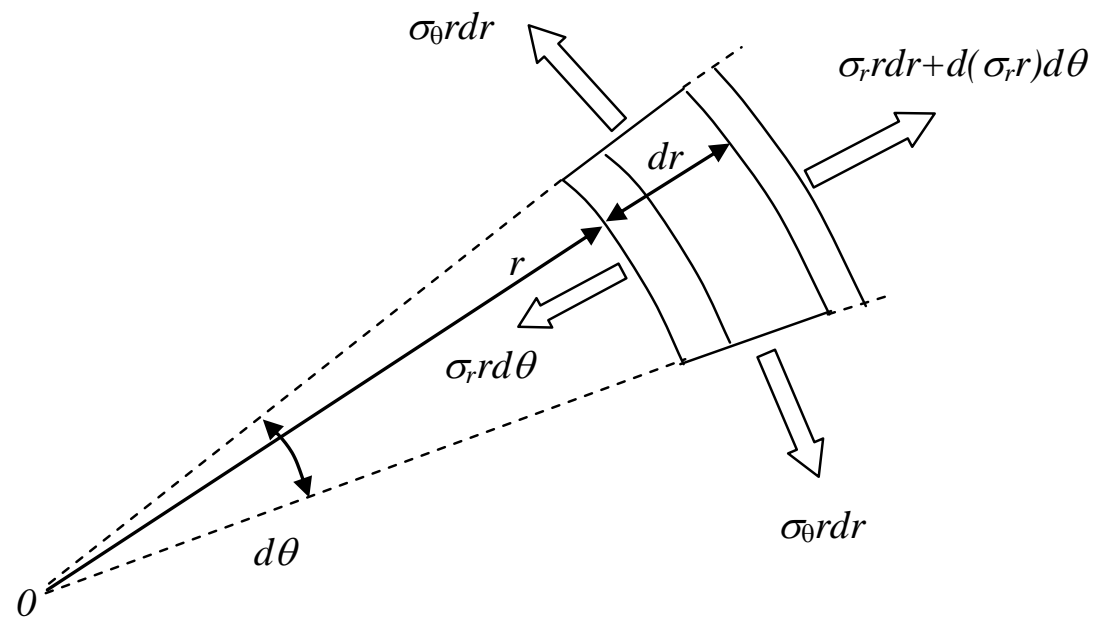

Рисунок 2. Напряжения и деформации в элементе трубы (рисунок автора)

Figure 2. Stresses and strains in the pipe member (the author's drawing)

Напряжения от изгиба суммируются по верхней образующей оболочки, и вычитаются по нижней образующей оболочки. Оболочка трубопровода может потерять устойчивость прямолинейной первоначальной формы равновесия и при действии сжимающих напряжений. При комбинированном воздействии изгиба и наружного давления необходимо проводить оценку прочности и устойчивости оболочки газопровода. Земли.

Около 90\% землетрясений в мире происходят наморском дне и только 10\% поверхности

при работе материала труб в упругой стадии следует суммировать с напряжениями от эксплуатационных нагрузок (давления, изменения температуры), учитывается, что все деформации (перемещения) от давления и температуры уже реализовались до приложения сейсмических нагрузок. [12].

Колебания частиц грунта вдоль продольной оси трубопровода изменяются по закону

$$
u=u_{0} \cos \frac{2 \pi}{T}\left(t-\frac{x}{C}\right),
$$

где: $u_{0}$ - максимальная амплитуда колебаний частиц грунта; $C$ - скорость сейсмической волны; $T$ - период колебаний.

При повороте трубопровода направление распространения сейсмической волны составит с осью трубопровода некоторый угол $\theta$.

В этом случае амплитуда смещения $u_{0}$ и длина волны заменяются соответственно на $u_{0} \sin \theta, u_{0} \cos \theta$ и $L_{0} / \sin \theta, L_{0} / \cos \theta L_{0}$ в (5) и (6).

Относительные продольные деформации и деформации изгиба трубопровода определяются по формулам 


$$
\begin{gathered}
\left|\varepsilon_{x}\right|=\left|2 \pi \frac{1}{C T} u_{0} \sin \frac{2 \pi}{T}\left(t-\frac{x}{C}\right)\right| \\
\left|\varepsilon_{и}\right|=\left|2 \pi^{2} \frac{1}{(C T)^{2}} B u_{0} \cos \frac{2 \pi}{T}\left(t-\frac{x}{C}\right)\right|
\end{gathered}
$$

где $D$ - наружный диаметр трубы.

Осевые (продольные) деформации трубопровода пропорциональны скорости колебания частиц грунта в сейсмической волне, а деформация изгиба - ускорению колебаний частиц грунта в сейсмической волне.

Для предотвращения местного смятия необходимо ограничить уровень изгибных деформаций в сечении трубопровода. При проверке на коллапс и при особом сочетании нагрузок морского трубопровода учитывается минимальная овализация $f_{0}=0,5 \%$, [3 табл. 9.14 и $9.15,4]$. (7).

В нормах [3] приведена формула определения овальности трубы от деформаций изгиба

$$
f^{\prime}{ }_{0}=\frac{f_{0}+\left\lfloor 0,030\left(1+\frac{D}{120 t}\right)\left(2 \varepsilon_{c} \frac{D}{t}\right)^{2}\right\rfloor}{1-\frac{P_{e}}{P_{c}}}
$$

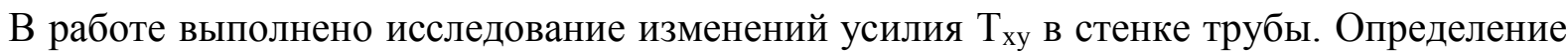
напряженно-деформированного состояния трубопровода выполнено с помощью программного комплекса «Лира» R9.4.7.

Параметром, влияющим на изменение жесткости, является изменение толщины стенок трубопровода. Но следует отметить, ее влияние на местную потерю устойчивости незначительно [3, 5].

Снижение жесткости около сварного шва приводит к возникновению деформаций, и увеличению изгиба трубы рядом со сварным швом.

Во всех нормативных методиках проектирования [3, 5, 10, 11] регламентируется значением критической изгибной деформации $\varepsilon_{\mathrm{c}}[4,11]$.

Упругая часть диаграммы момент-кривизна, может быть построена исходя из соотношения

$$
C=\frac{M}{E I}=\frac{M}{E \pi r^{3} d}
$$

где $C$ - кривизна трубы; изгибающий момент $M$ при изгибе С.

Упругопластическая часть диаграммы момент-кривизна в может быть получена на основании расчета моментов [6].

Данные для построения диаграммы «момент-кривизна» представлены в таблице 1.

Параметры построения диаграммы «момент-кривизна» морских металлических трубопроводов. 
Таблица 1/Table 1

Данные для построения диаграммы «момент-кривизна»/Data for construction of a «moment-curvature» diagram

\begin{tabular}{|c|c|c|c|}
\hline $\begin{array}{c}\text { № } \\
\text { п/п }\end{array}$ & Типоразмеры труб & \multicolumn{2}{|c|}{ Критерии проверки сейсмостойкости морских металлических } \\
трубопроводов.
\end{tabular}

При численном исследовании напряженно-деформированного состояния (НДС) морских трубопроводов, рассматривались следующие типоразмеры труб: $\varnothing 711 \times 25,4$ мм и $\varnothing 406$ × 17,5 мм. Данные результаты представлены на рис. 3 .

Для бетонного покрытия заводом-изготовителем указаны следующие характеристики [5].

Таблица 2/Table 2

Характеристики бетонного покрытия (сопротивление сдвига, прочность на сдвиг, прочность на сжатие)/Characteristics of concrete coating (shear resistance, shear strength, compression resistance)

\begin{tabular}{|c|c|c|}
\hline Характеристики бетонного покрытия & Значение & Ед. измерения \\
\hline Сопротивление сдвигу & 0,6 & кг $/ \mathrm{cm}^{2} ;-6 \mathrm{~T} / \mathrm{M}^{2}$ \\
\hline Прочность на сдвиг & 0,12 & МПа; 12,23 т/м \\
\hline Прочность на сжатие & 0,3 & МПа, $30 \mathrm{~T} / \mathrm{M}^{2}$. \\
\hline
\end{tabular}

Исследования НДС стенки трубы проводилось на сочетание эксплуатационных нагрузок (внутреннего давления продукта, температуры транспортировки продукта) и сейсмического

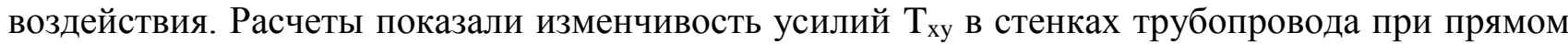
динамическом расчете по акселерограмме землетрясения [4].

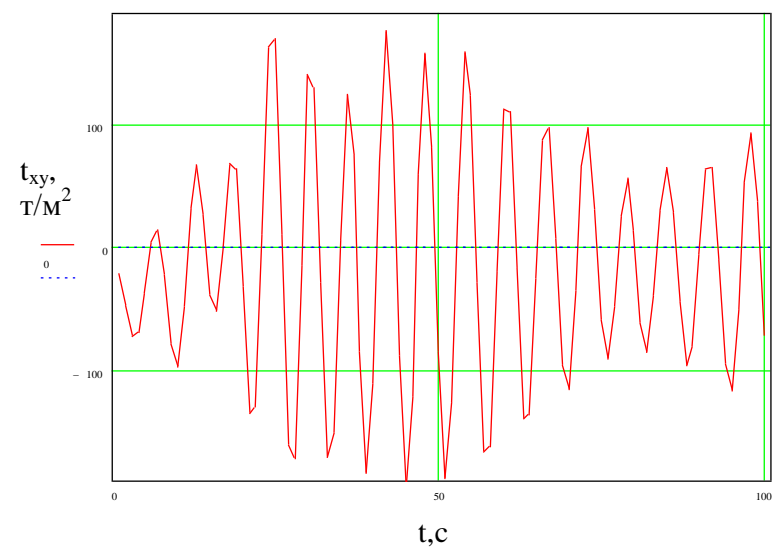

Рисунок 3. Изменение касательных напряжений $T_{x y}$ в стенке трубопровода при сейсмическом воздействии (рисунок автора)

Figure 3. Change of the shear stresses Txy in the pipe wall under a seismic load (the author's drawing) 
Главные напряжения в оболочке определяются исходя из следующих соотношений: $N_{1,2}=\frac{N_{x}+N_{z}}{2} \pm\left(\left(\frac{N_{x}+N_{z}}{2}\right)^{2}+T_{x y}^{2}\right)^{1 / 2}$

где: $N_{x}^{H^{\prime / B}}=N_{x} \pm 6 \frac{M_{x}}{h^{2}}, \quad N_{y}^{H / B}=N_{y} \pm 6 \frac{M_{y}}{h^{2}} \quad T_{x y}^{H / B}=T_{x y} \pm 6 \frac{{ }^{x y}}{h^{2}}-$ осевые напряжения на нижней и верхней поверхностях оболочки, $\mathrm{h}$ - толщина стенки трубопровода.

В нормативных документах $[8,9]$ отмечено, что касательные напряжения обычно не оказывают влияния на пластическое состояние оболочки и ими пренебрегают. Резкое падение жесткости около в сварном шве, приводит деформациям, к увеличению изгиба трубы рядом со сварным швом.

Сравнение расчета двух вариантов решения морских подводных трубопроводов без балластного бетонного покрытия и с бетонным покрытием показывает, что в трубопроводе без бетонного покрытия влияние землетрясения характеризуются большей изменчивостью выходных параметров напряженно-деформированного состояния труб ( $\left.\mathrm{N}_{\mathrm{x}}, \mathrm{T}_{\mathrm{xy}}, \mathrm{M}_{\mathrm{xy}}, \mathrm{Q}_{\mathrm{y}}\right)$ рис. 4.

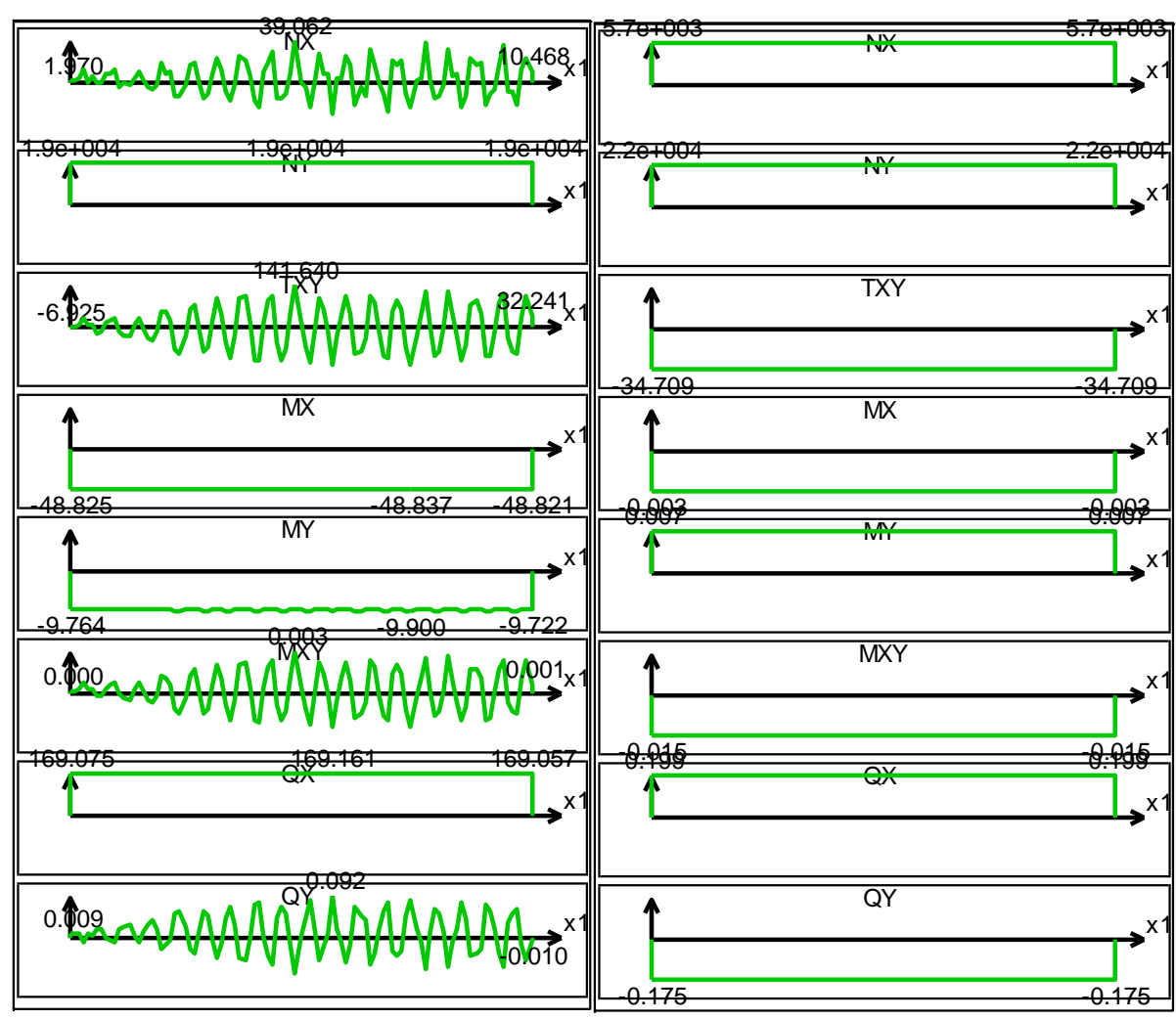

$\mathrm{a}$

6

Рисунок 4. Изменение напряжений $T_{x y}$ в стенке трубопровода ( $а$ - без бетонного покрытия, $\sigma$ - с бетонным покрытием) (рисунок автора)

Figure 4. Change of the stresses Txy in the pipe wall (a - without of a concrete coating, $b$ - with a concrete coating) (the author's drawing)

Наличие обетонирования снижает уровень и изменчивость касательных напряжений в внешней поверхности обетонированной трубы, но уровень касательных напряжений на верхней поверхности металлической трубы под обетонированием, отличается большим перепадом 


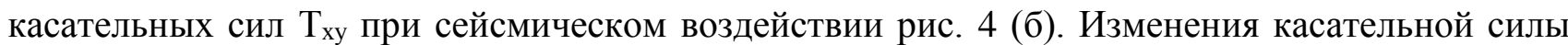
при контакте бетонного покрытия с изоляцией трубы может привести к трещинообразованию бетонного покрытия (рис. 5).

Изменение касательной силы Тху в металле трубопровода с бетонным покрытием, т/м2

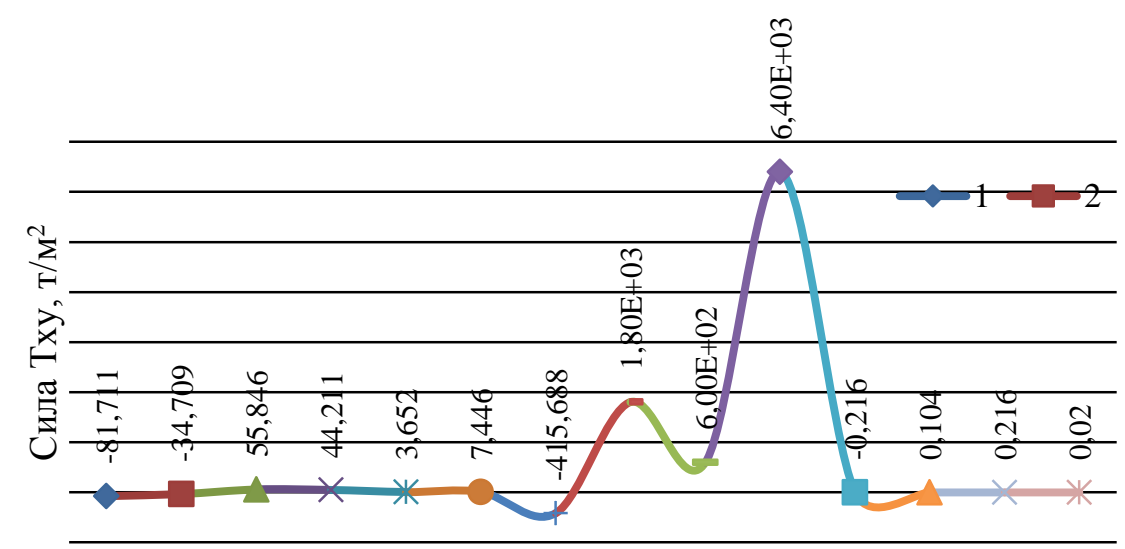

Протяженность участка трубопровода, м

Рисунок 5. Исследование изменений касательной силь $T_{x y}$ в стенке трубопровода с бетонным покрытием (рисунок автора)

Figure 5. Research of a tangential force Txy changes in the pipe wall with a concrete coating (the author's drawing)

В статье исследовано влияние сейсмических нагрузок и технологических нагрузок на эквивалентные напряжения, действующих в стенке подводного трубопровода.

Сопоставление данных характеристик табл. 2 с результатами конечно-элементного расчета на сейсмостойкость показывает, большую вероятность разрушения бетонного покрытия трубопровода при сейсмическом воздействии.

Наличие толстого и относительно жесткого бетонного покрытия с зазорами у монтажных швов, усиливает концентрацию напряжений вследствие измения жесткости в районе сварных швов. 


\section{ЛИТЕРАТУРА}

1. А.М. Кац, Теория упругости / А.М. Кац. - Санкт-Петербург: Издательство Лань, 2002. - $208 \mathrm{c}$.

2. ГОСТ Р 54382-2011, Нефтяная и газовая промышленность. Подводные трубопроводные системы / Москва, Стандартинформ, 2012. - 273 с.

3. Норвежский стандарт OS-F101 (Submarine pipeline systems. General requirements), Det Norske Veritas AS, 2013. - 272 c.

4. Отчет о научно-исследовательской работе по договору № PC-13/2015/203-03503 «Разработка требований к сейсмостойкости стальных морских подводных трубопроводов с повреждениями», Санкт-Петербург: СПГПУ, 2015. - 35 с.

5. ОТЧЁТ по результатам испытаний по определению упругого изгиба стальной трубы диаметром 820 мм, толщиной стенки 9 мм с наружным утяжеляющим бетонным покрытием (24.05.2016) «ЗУБ»- Астрахань: ООО «БТ СВАП», 2016. $16 \mathrm{c}$.

6. Правила классификации и постройки морских подводных трубопроводов: Российский морской регистр судоходства - Санкт-Петербург: Российский морской регистр судоходства, 2012. - 283 с.

7. Влияние сопротивления внешней среды на сейсмостойкость морских трубопроводов / Л.В. Муравьева. Труды RAO/GIS Offshore 20015.СПб.15-18 сентября 2015 // Сборник трудов 12-международной конференции по освоению ресурсов Российской Арктики и континентального шельфа стран CHГ RAO/CIS Offshore 2015.

8. Л.В. Муравьева, Безопасность и долговечность трубопроводных конструкций при динамических воздействиях / Л.В. Муравьева LAP LAMBERT Academic Publishing, 2012, 252 p.

9. Проектирование стальных конструкций. Часть 1-6. Прочность и устойчивость оболочек, ТКП ЕN 1993-1-6-2009, European Standard. 2010 г. 172 ст.

10. EN 1993-4-3: 2007 (E), Design of steel structures - Part 4-3, Pipelines. EN 1993-4Brussels: European committee for standardization, 2007, - 43 p.

11. BS EN 14161:2003. Petroleum and natural gas industries - Pipeline transportation systems (The European Standard EN 14161:2003 has the status of a British Standard), British standard, 2004, - 38 p.

12. Recommended Practice DNV-RP-F105 "Free Spanning Pipeliners", Det Norske Veritas, 2002. - 39 p. 
Muravyeva Liudmila Viktorovna

St. Petersburg state polytechnical university, Russia, St. Petersburg E-mail: rfludmia@yandex.ru

\title{
The impact of the shear stress change in the pipe body of sea-line with a concrete coating under a seismic load
}

\begin{abstract}
The thick-walled shell of the underwater pipeline experiences a combination of actions of the operational loads (pressure, temperature, weight of concrete coating), taking into account the probability of occurrence of vibration and seismic loads. The strength assessment of thick-walled shell of the pipeline must be done based on the stability check under the combined effect of bending and external pressure. The work objective is to study the special characteristics of the stress-strain state of the underwater pipeline sections with a concrete coating under the seismic loading.
\end{abstract}

Keywords: thick-walled shell; concrete cover; tangential force; earthquake resistance

\section{REFERENCES}

1. A.M. Kats, Teoriya uprugosti / A.M. Kats. - Sankt-Peterburg: Izdatel'stvo Lan', 2002. $208 \mathrm{~s}$.

2. GOST R 54382-2011, Neftyanaya i gazovaya promyshlennost'. Podvodnye truboprovodnye sistemy / Moskva, Standartinform, 2012. - 273 s.

3. Norvezhskiy standart OS-F101 (Submarine pipeline systems. General requirements), Det Norske Veritas AS, 2013. - $272 \mathrm{~s}$.

4. Otchet o nauchno-issledovatel'skoy rabote po dogovoru № RS-13/2015/203-03503 «Razrabotka trebovaniy $\mathrm{k}$ seysmostoykosti stal'nykh morskikh podvodnykh truboprovodov s povrezhdeniyami», Sankt-Peterburg: SPGPU, 2015. - 35 s.

5. OTChET po rezul'tatam ispytaniy po opredeleniyu uprugogo izgiba stal'noy truby diametrom $820 \mathrm{~mm}$, tolshchinoy stenki $9 \mathrm{~mm}$ s naruzhnym utyazhelyayushchim betonnym pokrytiem (24.05.2016) «ZUB»- Astrakhan': OOO «BT SVAP», 2016. - 16 s.

6. Pravila klassifikatsii i postroyki morskikh podvodnykh truboprovodov: Rossiyskiy morskoy registr sudokhodstva - Sankt-Peterburg: Rossiyskiy morskoy registr sudokhodstva, 2012. - $283 \mathrm{c}$.

7. Vliyanie soprotivleniya vneshney sredy na seysmostoykost' morskikh truboprovodov / L.V. Murav'eva. Trudy RAO/GIS Offshore 20015.SPb.15-18 sentyabrya 2015 // Sbornik trudov 12-mezhdunarodnoy konferentsii po osvoeniyu resursov Rossiyskoy Arktiki i kontinental'nogo shel'fa stran SNG RAO/CIS Offshore 2015.

8. L.V. Murav'eva, Bezopasnost' i dolgovechnost' truboprovodnykh konstruktsiy pri dinamicheskikh vozdeystviyakh / L.V. Murav'eva LAP LAMBERT Academic Publishing, 2012, 252 p.

9. Proektirovanie stal'nykh konstruktsiy. Chast' 1-6. Prochnost' i ustoychivost' obolochek, TKP EN 1993-1-6-2009, European Standard. 2010 g. 172 st.

10. EN 1993-4-3: 2007 (E), Design of steel structures - Part 4-3, Pipelines. EN 1993-4Brussels: European committee for standardization, 2007, - 43 p.

11. BS EN 14161:2003. Petroleum and natural gas industries - Pipeline transportation systems (The European Standard EN 14161:2003 has the status of a British Standard), British standard, 2004, - 38 p.

12. Recommended Practice DNV-RP-F105 "Free Spanning Pipeliners", Det Norske Veritas, 2002. - $39 \mathrm{p}$. 